\title{
Evaluation of abdominal fat index by ultrasonography and its relationship with psoriasis and metabolic syndrome
}

\author{
Müzeyyen Gönül' ${ }^{1}$ İdil Tatar², Filiz Canpolat ${ }^{1}$, Gökçe Işıl Kurmus ${ }^{1}$ Can Ergin ${ }^{1}$, Baki Hekimoğlu² \\ ${ }^{1}$ Department of Dermatology, Dışkapı Yıldırım Beyazıt Education and Research Hospital, Ankara, Turkey \\ 2Department of Radiology, Dışkapı Yıldırım Beyazıt Education and Research Hospital, Ankara, Turkey \\ Adv Dermatol Allergol 2017; XXXIV (5): 453-456 \\ DOI: https://doi.org/10.5114/ada.2017.71111
}

\begin{abstract}
Introduction: Accumulating evidence indicates that psoriasis is associated with obesity and metabolic syndrome. Psoriasis and obesity share similar inflammatory mediators, and obesity may potentiate some inflammatory cytokines seen in psoriasis. Body fat distribution, particularly visceral adipose tissue (VAT), is an important factor in metabolic syndrome and atherosclerotic diseases. An association has been demonstrated between psoriasis and abdominal VAT measured by computed tomography (CT).

Aim: To measure abdominal VAT noninvasively by ultrasonography (USG) in patients with psoriasis and investigated its relation to psoriasis and metabolic syndrome.

Material and methods: The study population consisted of 41 psoriasis patients and 41 control subjects matched for age, sex, and body mass index. The maximal preperitoneal fat thickness (Pmax) at the anterior surface of the liver and the minimal subcutaneous fat thickness (Smin) of the abdomen were measured by USG. The abdominal fat index (AFI = Pmax/Smin ratio) was calculated and the results were compared between groups.

Results: The rate of metabolic syndrome was significantly higher in psoriasis patients $(p=0.0018)$. The mean AFI was similar in both groups. AFI was not associated with psoriasis in subjects with metabolic syndrome $(p=0.495)$ or with Psoriasis Area and Severity Index $(r=0.123, p=0.443)$.

Conclusions: This is the first study to evaluate abdominal VAT by USG. Computed tomography may be more reliable than USG, but its high cost and radiation exposure are major disadvantages. Further studies are required to determine the relationships between psoriasis and VAT.
\end{abstract}

Key words: psoriasis, metabolic syndrome, ultrasonography, visceral adipose tissue.

\section{Introduction}

Psoriasis is a chronic inflammatory skin disease affecting the skin, nails, and joints. The accumulating evidence has shown that psoriasis is not an organ-specific disease, but instead is a systemic inflammatory disease associated with cardiovascular diseases and metabolic syndrome (MS), consisting of obesity, hypertension, dyslipidemia, and insulin resistance [1-3].

Obesity is a heterogeneous condition, and not all obese patients have comorbidities. Body fat distribution is an important factor in both MS and atherosclerotic diseases [4]. Particularly, visceral adipose tissue (VAT) is more closely related to metabolic disturbances and atherosclerotic diseases than subcutaneous or total body fat [4]. The VAT has been shown to contribute to insulin resistance, coronary artery disease, and carotid atherosclerosis [4]. Adipose tissue not only stores energy but also functions as an endocrine organ that secretes various bioactive substances referred to as adipokines [5]. Dysregulation of these factors occurs due to excess adiposity, and adipocyte dysfunction contributes to the pathogenesis of various diseases via altered immune responses [5]. Thus, obesity is accepted as a chronic lowgrade inflammatory state and it has been reported to share similar mediators of inflammation with psoriasis and to contribute to inflammatory processes in psoriasis $[6,7]$.

In a recent study, Balci et al. examined abdominal fat distribution in psoriasis patients by computed tomography (CT) [8]. Visceral fat area (VFA: fat tissue in the intra-

Address for correspondence: Can Ergin, Department of Dermatology, Dışkapı Yıldırım Beyazıt Education and Research Hospital, Yeşilyurt Sok. No: 15/7 Kavaklıdere, 06690 Ankara, Turkey, phone: +90 5056831264, e-mail: drcanergin@hotmail.com Received: 17.02.2016, accepted: 22.06.2016. 
peritoneal, omental, and retroperitoneal areas) and subcutaneous fat area (SFA) were measured. They described the relation between VFA, MS, and psoriasis.

\section{Aim}

In this study, we aimed to measure VAT by ultrasonography (USG), a cheaper and safer imaging method, to evaluate the associations between VAT, MS, and psoriasis.

\section{Material and methods}

A total of 41 patients with clinically and histopathologically diagnosed psoriasis and 41 age-, sex-, and body mass index (BMI)-matched controls were enrolled in this study. Informed consent was obtained from all participants and the local ethics committee approved the study. The severity of psoriasis was quantified using the Psoriasis Area and Severity Index (PASI). Controls were recruited from among patients with warts, tinea pedis, or melanocytic nevi without any inflammatory disease. Patients that had received systemic or topical treatment for psoriasis within the last month were excluded. Participants younger than 18 years or with any renal disease, metabolic disorder, or endocrine disease apart from diabetes and/or obesity and receiving antihyperlipidemic agents were also excluded.

Age, sex, weight, height, BMI, disease duration, medications and smoking habits were recorded. Waist and hip circumferences, waist-to-hip ratio (WHR), and blood pressure were noted. Peripheral venous blood samples were collected in the morning after an 8-hour fast. Tota cholesterol, high-density lipoprotein (HDL), low-density lipoprotein (LDL), triglyceride, liver enzyme, fasting blood glucose, and insulin levels were measured. The criteria of the National Cholesterol Education Program's Adult Panel III were used to diagnose MS. These criteria include: 1) waist circumference $>102 \mathrm{~cm}$ in men or $>88 \mathrm{~cm}$ in women; 2) hypertriglyceridemia ( $\geq 150 \mathrm{mg} / \mathrm{dl}$ ); 3) $\mathrm{HDL}$ cholesterol $<40 \mathrm{mg} / \mathrm{dl}$ in men or $<50 \mathrm{mg} / \mathrm{dl}$ in women; 4) arterial blood pressure $\geq 130 / 85 \mathrm{~mm} \mathrm{Hg}$ or antihypertensive medication; and 5) fasting plasma glucose level $\geq 110 \mathrm{mg} / \mathrm{dl}$ or known diabetes mellitus. Subjects positive for three or more of these criteria were diagnosed with MS [9].

USG was performed by the same radiologist after an overnight fast, and the maximal thickness of preperitoneal fat (Pmax) at the anterior surface of the liver and the minimal thickness of subcutaneous fat (Smin) in the upper median abdomen were measured. The abdominal fat index value (AFI = Pmax/Smin ratio), which is an indicator of visceral fat deposition and may reflect metabolic disorders such as hyperlipidemia and glucose intolerance [10], was then calculated.

\section{Statistical analysis}

IBM SPSS Statistics 21.0 was used for the statistical analysis. Chi-square $\left(\chi^{2}\right)$ test was used to compare categorical parameters. Independent Samples $t$ test or One-way ANOVA test were used to compare the mean values of the different parameters of the groups. Pearson's correlation test was used to analyze the correlations between the independent variables.

\section{Results}

The study group consisted of 22 males and 19 females, and the control group consisted of 21 males and 20 females. The demographic features, clinical findings, and laboratory test results are shown in Table 1 . The mean PASI of the patients ranged from 2.7 to 30.5. Only two patients had PASI below 5. So most of the patients had moderate or severe psoriasis. There were no significant differences between the study and control groups in terms of age, sex, BMI, WHR, diastolic blood pressure, serum fasting blood glucose, insulin, total cholesterol, LDL cholesterol, HDL cholesterol, triglyceride level, or AFI. In contrast, the mean systolic blood pressure was significantly higher in the study group $(p=0.007)$. The HDL cholesterol levels of the psoriasis patients were lower $(p=0.073)$ and the insulin levels of the study group were higher ( $p=0.069$ ) than those of the control subjects, but the differences were not statistically significant.

A total of 18 of 41 psoriasis patients (43.9\%) and 8 of 41 (19.5\%) controls had MS, and this difference was statistically significant ( $p=0.018)$. The AFI values did not differ between MS patients with psoriasis and those without psoriasis $(p=0.443)$. The AFI was not correlated with the PASI $(r=-0.123, p=0.443)$.

The mean PASI of the psoriasis patients with MS $(n=$ $18)$ and without MS $(n=23)$ were $13.0 \pm 7.6$ and $12.0 \pm 7.3$, respectively, and the values of both groups were comparable to each other $(p=0.665)$. The mean AFI of the patients with MS $(n=18)$ and without MS $(n=23)$ were $1.20 \pm 0.84$ and $1.20 \pm 0.67$, respectively, and the difference was not significant between the groups ( $p=0.495)$.

There were no statistically significant correlations between AFI and BMI $(r=-0.128, p=0.25)$, WHR $(r=0.030$, $p=0.78)$, fasting blood glucose $(r=0.156, p=0.38)$, systolic blood pressure $(r=0.099, p=0.37)$, diastolic blood pressure $(r=-0.1, p=0.16)$, serum total cholesterol $(r=$ $0.141, p=0.2)$, or triglyceride levels $(r=0.024, p=0.83)$.

The mean AFI of the psoriasis patients who smoked $(n=27, \mathrm{AFI}=1.21)$ was significantly higher than those of the patients who did not smoke $(n=14, \mathrm{AFI}=0.78)$ $(p=0.009)$.

\section{Discussion}

Psoriasis is associated with increased rates of various comorbidities, including arterial hypertension, dyslipid- 
Table 1. Demographic, clinical features and laboratory results of the subjects

\begin{tabular}{lccc}
\hline Parameter & Patients $(n=41)$ & Controls $(n=41)$ & $P$-value \\
\hline Male/female & $22 / 19$ & $21 / 20$ & 0.825 \\
\hline Age [year] & $43.2 \pm 15.6$ & $41.3 \pm 14.8$ & $0.584^{*}$ \\
\hline Disease duration [year] & $14.6 \pm 12.1$ & & \\
\hline PASI & $12.5 \pm 7.4$ & & $0.724^{*}$ \\
\hline BMI [kg/m² & $28.1 \pm 5.9$ & $27.7 \pm 5.3$ & $0.888^{*}$ \\
\hline Waist/hip ratio & $0.917 \pm 0.09$ & $0.914 \pm 0.09$ & $0.007^{*}$ \\
\hline BP systolic [mm Hg] & $127 \pm 14$ & $119 \pm 10$ & $0.659^{*}$ \\
\hline BP diastolic [mm Hg] & $75 \pm 9$ & $74 \pm 12$ & $0.487^{*}$ \\
\hline Fasting blood glucose [mg/dl] & $91.8 \pm 34.3$ & $87.7 \pm 15.7$ & $0.069^{*}$ \\
\hline Insulin & $13.3 \pm 8.2$ & $10.51 \pm 5.6$ & $0.656^{*}$ \\
\hline Total cholesterol [mg/dl] & $175.2 \pm 37$ & $171.5 \pm 37.9$ & $0.799^{*}$ \\
\hline LDL cholesterol [mg/dl] & $102.7 \pm 35.2$ & $104.6 \pm 31.3$ & $0.073^{*}$ \\
\hline HDL cholesterol [mg/dl] & $41 \pm 10.8$ & $45.4 \pm 11.4$ & $0.856^{*}$ \\
\hline Triglyceride [mg/dl] & $143.7 \pm 87.9$ & $40.1 \pm 90.1$ & $0.835^{*}$ \\
\hline Smin [mm] & $12.9 \pm 6.4$ & $13.2 \pm 6.6$ & $0.428^{*}$ \\
\hline Pmax [mm] & $13.2 \pm 5.3$ & $12.3 \pm 4.5$ & $0.928^{*}$
\end{tabular}

*Student's t-test. PASI - psoriasis area and severity index, BMI - body mass index, BP - blood pressure, Smin - minimal thickness of subcutaneous fat, Pmax - maximal thickness of preperitoneal fat, AFI-abdominal fat index.

emia, obesity, diabetes mellitus, and MS [11]. Obesity was demonstrated to be an independent risk factor for developing psoriasis [7, 12]. Obesity, a chronic inflammatory condition, causes increased levels of proinflammatory cytokines such as tumor necrosis factor $\alpha$ (TNF- $\alpha$ ) and interleukin 6 (IL-6) [7]. Chronic inflammation in psoriasis is additionally aggravated by obesity. Major adipokines, which are dysregulated in obesity, were also shown to be abnormal in psoriasis, and both conditions are assumed to share similar immunological mechanisms [13].

Adipose tissue is not only involved in energy storage; it is also an endocrine organ that contributes to immune responses [7]. Adipose tissue, which consists of adipocytes, endothelial cells, leukocytes, fibroblasts, and macrophages, plays prominent roles in various metabolic processes, local and systemic inflammation, vascular function, and immune regulation by secreting adipokines and cytokines, including adiponectin, leptin, resistin, visfatin, retinol-binding protein 4 , omentin and TNF- $\alpha[7$, 14]. Bioactive substances, including IL-1 $\beta$, IL-6, IL-8, IL$17, \mathrm{IL}-18$, and plasminogen activator inhibitor are also synthesized by adipose tissue [14]. All of these bioactive substances, which play roles in MS, insulin resistance, and psoriasis, are secreted by VAT [8]. Therefore, VAT is more closely related to metabolic and atherosclerotic disturbances than subcutaneous and/or total body fat [8].

In a recent study, Balci et al. [8] evaluated the increased visceral fat level in patients with psoriasis and its contribution to MS. In this study, VFA was shown to be increased in psoriasis patients and to be independently associated with the presence of psoriasis. It was also shown that VFA was independently associated with the presence of MS in psoriasis. In conclusion, it was speculated that increased VAT was a major contributor to metabolic and cardiovascular comorbidities associated with psoriasis by releasing proinflammatory cytokines and adipokines [8]. Balci et al. [8] measured VAT by $\mathrm{CT}$, which is both expensive and exposes patients to radiation. In another study, AFI was shown to be most closely correlated with the VFA/SFA ratio measured by CT [10]. Furthermore, USG was demonstrated to be more accurate than anthropometric measurements for measuring intraabdominal fat [15]. Therefore, we used USG to measure VAT.

The results of our study were different from those of Balci et al. [8]. We found no significant difference in AFI between patients with psoriasis and controls, there were no correlations between AFI and the components of MS, and the presence of psoriasis was not an independent risk factor for $\mathrm{AFI}$ in patients with MS. In addition, we found a strong association between AFI and smoking. Chatkin et al. obtained similar results [16].

Balci et al. [8] included patients with psoriasis who had been receiving systemic treatment in the patient group, and their control group included patients with inflammatory or immune-related diseases such as lichen 
planus, urticaria, and contact dermatitis. These conditions may have interfered with the reliability of their study. In our study, patients that had been receiving systemic or topical treatment for psoriasis for the last month and those with inflammatory disorders were excluded from the analysis.

We think that the cellular composition or altered functions of VAT or other factors rather than the amount of VAT may make a more significant contribution to MS and/or psoriasis. A recent study supported our hypothesis and suggested that the pathogenic potential of visceral adipocyte hypertrophy usually requires the limitation and/or impairment of other body organs in predisposed patients [17]. Another possible explanation for the discrepancy between our results and those of Balci et al. [8] is that USG may not be as precise as CT for the measurement of visceral fat thickness.

\section{Conclusions}

Large placebo-controlled studies are required to determine the interactions between VAT and psoriasis. We think that USG may be an alternative, cheap and safe method for measuring VAT, because CT has the major disadvantages of radiation exposure, high cost, and technical difficulty.

\section{Conflict of interest}

The authors declare no conflict of interest.

\section{References}

1. Sales R, Torres T. Psoriasis and metabolic syndrome. Acta Dermatovenerol Croat 2014; 22: 169-74.

2. O'Neill S, O'Driscoll L. Metabolic syndrome: a closer look at the growing epidemic and its associated pathologies. Obes Rev 2015; 16: 1-12.

3. Bangemann K, Schulz W, Wohlleben J, et al. Depression and anxiety disorders among psoriasis patients: protective and exacerbating factors. Hautarzt 2014; 65: 1056-61.

4. Kobayashi H, Nakamura T, Miyaoka K, et al. Visceral fat accumulation contributes to insulin resistance, small-sized low density lipoprotein, and progression of coronary artery disease in middle-aged non-obese Japanese men. Jpn Circ J 2001; 65: 193-9.

5. Al-Suhaimi EA, Shehzad A. Leptin, resistin and visfatin: the missing link between endocrine metabolic disorders and immunity. Eur J Med Res 2013; 18: 12.

6. Ouchi N, Parker JL, Lugus JJ, Walsh K. Adipokines in inflammation and metabolic disease. Nat Rev Immunol 2011; 11: 85-97.

7. Gerdes S, Rostami-Yazdi M, Mrowietz U. Adipokines and psoriasis. Exp Dermatol 2011; 20: 81-7.

8. Balci A, Balci DD, Yonden Z, et al. Increased amount of visceral fat in patients with psoriasis contributes to metabolic syndrome. Dermatology 2010; 220: 32-7.

9. Executive Summary of The Third Report of The National Cholesterol Education Program (NCEP) Expert Panel on Detection, Evaluation, And Treatment of High Blood Cholestero
In Adults (Adult Treatment Panel III). Expert Panel on Detection, Evaluation, and Treatment of High Blood Cholesterol in Adults. JAMA 2001; 285: 2486-97.

10. Suzuki R, Watanabe S, Hirai Y, et al. Abdominal wall fat index, estimated by ultrasonography, for assessment of the ratio of visceral fat to subcutaneous fat in the abdomen. Am Med 1993; 95: 309-14

11. Radtke MA, Mrowietz U, Feuerhahn J, et al. Early detection of comorbidity in psoriasis: recommendations of the National Conference on Healthcare in Psoriasis. J Dtsch Dermatol Ges 2015; 13: 674-89.

12. Herron MD, Hinckley M, Hoffman MS, et al. The impact of obesity and smoking on psoriasis presentation and management. Arch Dermatol 2005; 141: 1527-34.

13. Baran A, Flisiak I, Jaroszewicz J, Świderska M. Effect of psoriasis activity on serum adiponectin and leptin levels. Postep Dermatol Alergol 2015; 32: 101-6.

14. Gerkowicz A, Pietrzak A, Szepietowski JC, et al. Biochemical markers of psoriasis as a metabolic disease. Folia Histochem Cytobiol 2012; 50: 155-70.

15. Shabestari AA, Bahrami-Motlagh H, Hosseinpanah F, Heidari K. Abdominal fat sonographic measurement compared to anthropometric indices for predicting the presence of coronary artery disease. J Ultrasound Med 2013; 32: 1957-65.

16. Chatkin R, Chatkin JM, Spanemberg L, et al. Smoking is associated with more abdominal fat in morbidly obese patients. PLoS One 2015; 10: e0126146.

17. Bays HE. "Sick fat," metabolic disease, and atherosclerosis. Am J Med 2009; 122: 26-37. 